Southern California youth is a serious issue and frequently observed at relatively young age. The shift towards extreme body weights is likely to cause an enormous burden of adverse health outcomes once these youth grow older.

Keywords: Extreme Obesity in Southern California youth, Obesity treatment, Adolescent health outcomes

doi:10.3121/cmr.2010.943.ps2-23

\section{HMORN Infrastructure}

PS1-27:

Use of Tumor Registry to Obtain Family History Information

Kimberly Lane, $\mathrm{MPH}^{1,2}$; JeffreyBrown, $\mathrm{PhD}^{1,2}$; Richard Platt, $\mathrm{MD}, \mathrm{MSc}^{1,2}$

${ }^{1}$ Harvard Pilgrim Health Care Institute; ${ }^{2}$ Harvard Medical School

Background: The Human Genome project has revolutionized the field of medicine to move toward personalized medicine. The family history is a screening tool to identify families at risk for common conditions allowing for personalized care. For breast cancer syndromes, genetic testing, counseling and prophylactic treatment are available. While there are data about the efficacy and clinical utility of the family history, there are areas that still need investigation. Does obtaining the family history lead to patient recommendations? Do patients FDA's Mini-Sentinel Initiative: The HMORN's Role.

In May 2008, the FDA Commissioner announced FDA's intention to create the Sentinel Initiative. The Sentinel Initiative is an effort by the FDA to create a coordinated national electronic medical product safety surveillance system. The Food and Drug Administration Amendments Act of 2007 (FDAAA) mandated FDA develop methods to obtain access to disparate data sources and to establish an active post-market risk identification and analysis system linking and analyzing data from multiple sources. The law sets a goal of 25 million patients under surveillance by July 1, 2010, and 100 million patients by July 1, 2012. With the funding and support provided by FDAAA, the FDA laid out the vision for Sentinel Initiative. In September 2009, the FDA awarded a single contract to a consortium of 28 institutions led by the Harvard Medical School Department of Population Medicine at the Harvard Pilgrim Health Care Institute, including 13 HMORN members (all Kaiser Permanente plans, Group Health Cooperative, Lovelace Clinic Foundation, Myers Primary Care Institute, Marshfield Clinic Research Foundation, HealthPartners Research Foundation and Henry Ford Health Services), CIGNA, Humana, WellPoint [HealthCore] and Tennessee's Medicaid system, comprising $\sim 60$ million covered lives. Additional data resources include the HMORN's ambulatory electronic medical records linked to enrollment and claims data for $\sim 10$ million patients; inpatient electronic medical records (EMRs), including blood product use from 88 adult and pediatric hospitals (approximately half are Kaiser plans); plus device and disease registries. Mini-Sentinel will create a 'laboratory' for developing and evaluating safety surveillance scientific methodologies and offers FDA the opportunity to evaluate safety issues in existing automated healthcare data system(s) while learning about the barriers and challenges inherent in these activities. The collaboration provides FDA with a public-private solution that leverages the strengths of the private sector and academia. Over the next five years, this collaboration will work with FDA to create the foundation for the Sentinel Initiative's long-term goal of setting up a coordinated national post-market safety surveillance system.

Keywords: Tumor registry, Family history screening, Sentinel Initiative doi:10.3121/cmr.2010.943.ps1-27
PS1-28:

Kaiser Permanente Center for Effectiveness and Safety Research

Donald Bachman, $\mathrm{MS}^{1}$; Pierre-Andre La Chance, $\mathrm{BS}^{1}$; Mark Hornbrook, $\mathrm{PhD}^{1}$

${ }^{1}$ Kaiser Permanente Center for Health Research

Background: Kaiser Permanente has inaugurated an interregional research initiative focusing on comparative effectiveness and safety (CESR). All eight regions will partner to take advantage of their large and diverse populations, exceptional scientists, and extensive data resources. Aim: To provide HMORN collaborators: (1) an understanding of where CESR is and where it will be going; (2) a description of CESR governance rules; and, (3) a guide to pathways for partnerships with collaborators.

Keywords: Inter-regional research, Comparative effectiveness and safety doi:10.3121/cmr.2010.943.ps1-28

PS1-29:

Resources to Facilitate Multi-site Collaboration: the PRIMER Research Toolkit

Sarah Greene, $\mathrm{MPH}^{1}$; Ella Thompson, $\mathrm{BS}^{1}$; Laura-Mae Baldwin, MD, $\mathrm{MPH}^{2}$; Anne Victoria Neale, $\mathrm{PhD}, \mathrm{MPH}^{3}$; Rowena Dolor, $\mathrm{MD}, \mathrm{MHS}^{4}$

${ }^{1}$ Group Health Cooperative; ${ }^{2}$ University of Washington; ${ }^{3}$ Wayne State University School of Medicine; ${ }^{4}$ Duke University Medical Center

Background and Aims: The national research enterprise has typically functioned in a decentralized fashion, resulting in duplicative or undocumented processes, impeding not only the pace of research, but diffusion of established best practices. To remedy this, many long-standing networks have begun capturing and documenting proven strategies to streamline and standardize various aspects of the research process. The project, "Partnership-driven Resources to IMprove and Enhance Research" (PRIMER), was funded through the Clinical and Translational Science Awards (CTSA) initiative to leverage the collective expertise from two networks: the HMO Research Network and Practice Based Research Networks (PBRNs). Each network has a shared goal of propagating research resources and best practices. Methods: We created and distributed an online survey to 92 CTSA and PBRN representatives in March, 2009 to define critical needs and existing resources that could inform a resource repository. The survey identified barriers and benefits to forming research partnerships, and assessed the perceived utility of various tools that could accelerate the research process. The study team identified, reviewed and organized tools based on the typical research trajectory from design to dissemination. Results: Fifty-five of 92 invitees $(59 \%)$ completed the survey. Respondents rated the ability to conduct community-relevant research through true academic-community partnerships as the top-rated benefit of multi-site research, followed by the opportunity to accelerate translation of research into practice. The top two perceived barriers to multi-site research were 'funding opportunities are not adequate (e.g., too few, not enough to support true collaborations), and 'lack of research infrastructure to support [all] partners (e.g., no IT support, IRB, dedicated research staff). Respondents' ratings of the utility of various tools and templates was used to guide development of an online repository: www. ResearchToolkit.org, which is comprised of over 120 distinct resources. Conclusions: We are disseminating the ResearchToolkit website via academic and media channels, and identifying options for making it a sustainable resource. Given the dynamic nature of the research enterprise, maintenance and accuracy of a web-based resource is challenging. Still, the positive response to the toolkit suggests that there is high interest in sustaining it. We will demonstrate the Toolkit as part of this conference.

Keywords: Partnership-driven Resources to Improve and Enhance Research, Research processes, Forming research partnerships

doi:10.3121/cmr.2010.943.ps1-29 